\title{
Low cost, low pass Prism filtering
}

\author{
Manus Henry ${ }^{\mathrm{a}, \mathrm{b}}$ \\ aDepartment of Engineering Science \\ University of Oxford \\ Oxford, OX1 3PJ, UK. \\ manus.henry@eng.ox.ac.uk
}

\author{
${ }^{\mathrm{b}}$ School of Electrical Engineering and Computer Science \\ South Ural State University \\ Chelyabinsk, Russia
}

\begin{abstract}
Prism signal processing is a new FIR signal processing technique offering a fully recursive calculation and minimal design cost, which is well-suited to the requirements of IoT sensing. This paper describes a family of low pass filter designs using one or more layers of Prisms, where the frequency response has minimal stopband gain. Combined with heterodyning and Prism-based tracking, these filters provide flexible and powerful real-time tracking of signal component properties for use in measurement applications.
\end{abstract}

Keywords - Industrial cyber-physical systems, Recursive FIR filtering, Prism signal processing, signal tracking.

\section{INTRODUCTION}

Prism Signal Processing (PSP) [1] is a new FIR technique particularly suited to metrological tasks in the context of the Internet of Things (IoT) [2]. Measurement applications include pressure sensor validation [3], fast flow measurement [4, 5] and condition monitoring of rotating machinery $[6,7]$.

The Prism (Fig. 1) is an FIR filter generating one or two outputs. Its properties are defined by its characteristic frequency $m$ and harmonic number $h$ [1]. It consisting of two layers of cascaded integration blocks, wherein the input signal is multiplied by a modulating sinusoid of frequency $\mathrm{m} \times \mathrm{h} \mathrm{Hz}$ and integrated over a window of period $1 / \mathrm{m} \mathrm{s}$. It offers several desirable properties: the calculation is FIR, and hence robust; the outputs have linear phase delay; the calculation is fully recursive so that the computational cost per sample is low and fixed, irrespective of the length of the filter; and Prism design is straightforward, given desired values of $m$ and $h$, requiring only the evaluation of linearly spaced sine and cosine values.

The Prism's design simplicity and low computational cost facilitate the use of Prism networks to carry out a variety of signal processing tasks, including bandpass and notch filtering, and tracking [2], whereby frequency/phase/ amplitude values are calculated for an input signal.

For example, Refs $[8,9]$ describe a PSP bandpass filter design, including an FPGA implementation with a real-time performance which would require supercomputer resources if calculated using conventional FIR convolution. Here we describe a family of low pass filter designs with minimal stopband gains. These offer tradeoffs between stopband attenuation, computational load and dynamic response. A simulation demonstrates good tracking of frequency/ phase/amplitude values for a noisy signal component with a large and adjacent undesired frequency component.

\section{FILTER DESIGN}

For the single output Prism of Fig. 1, the computational cost of update the output $G_{c}{ }^{h}(t)$, after receiving a new sample at the input $s(t)$, is 12 multiplications and 22 additions, irrespective of the length of the filter window [2]. Accordingly, elaborate networks of Prisms can be constructed to provide designed signal characteristics which remaining computationally viable.

Fig. 2 shows the frequency response of the Prism output $G_{s}{ }^{h}$ for $h=1,2,3$. The response for $h=1$ has the highest peak and fastest attenuation, but still has significant gain above $m$. The design aim for creating a 'low pass' filter is to use a combination of Prisms to minimise the maximum gain for all frequencies above $m$. This is achieved using one or more Prism layers, as illustrated in Fig. 3. Three Prisms share the same input signal $s(t)$ and value of $m$, but have distinct values $h=1,2,3$. Their outputs are combined to produce the layer output $L(t)$ using weights $w_{1}, w_{2}, w_{3}$, which are selected to minimize the maximum gain above $m$. Note that with common $m$ the Prisms have identical (linear) phase delay, so combining their outputs affects the gain, but not the phase, of the output.

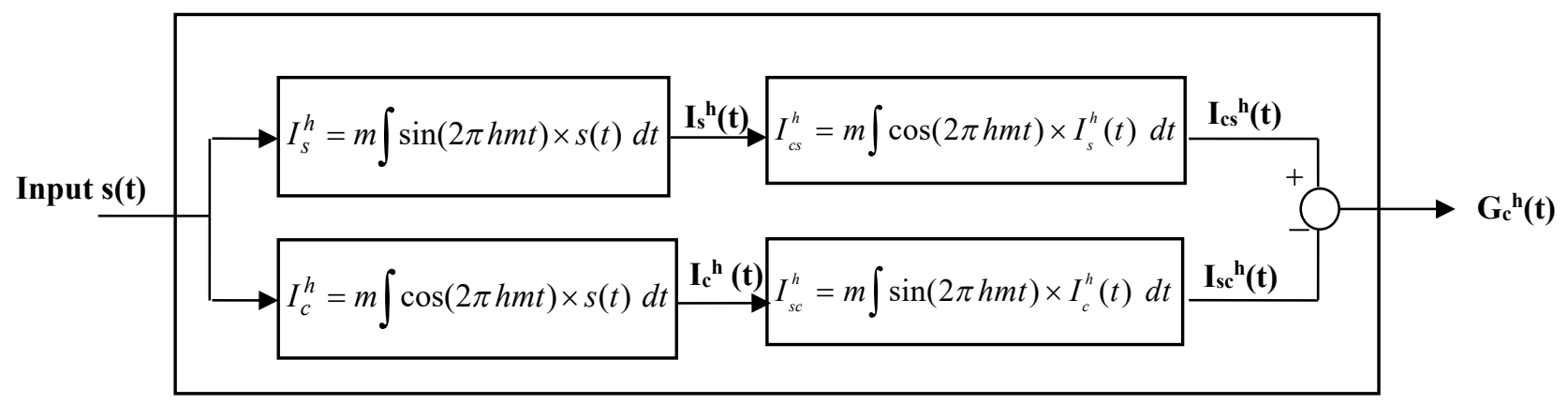

Fig. 1. Structure of Prism to generate a single output, $G_{c}{ }^{h}$. 

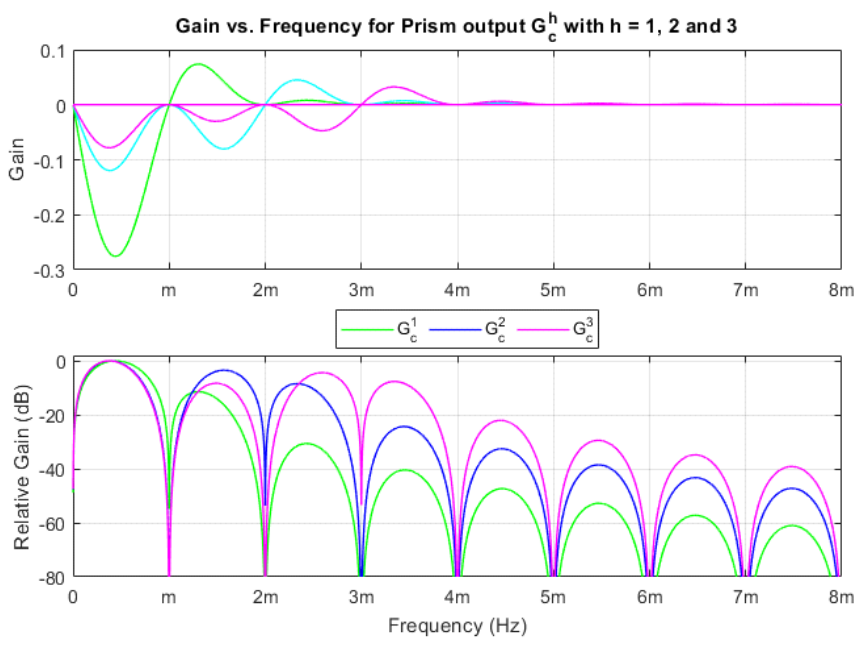

Fig. 2. Gains of Prism output $G_{c}{ }^{h}$ for $h=1,2$ and 3 .

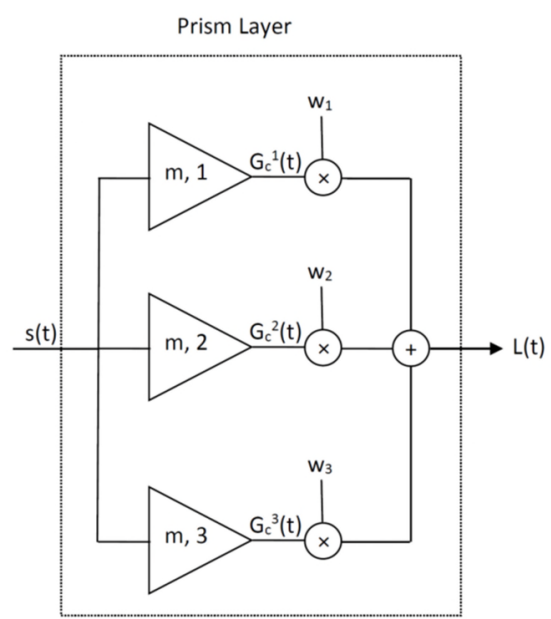

Fig. 3. Prism layer. Each Prism is represented as a triangle labelled with its corresponding values of $m$ and $h$.

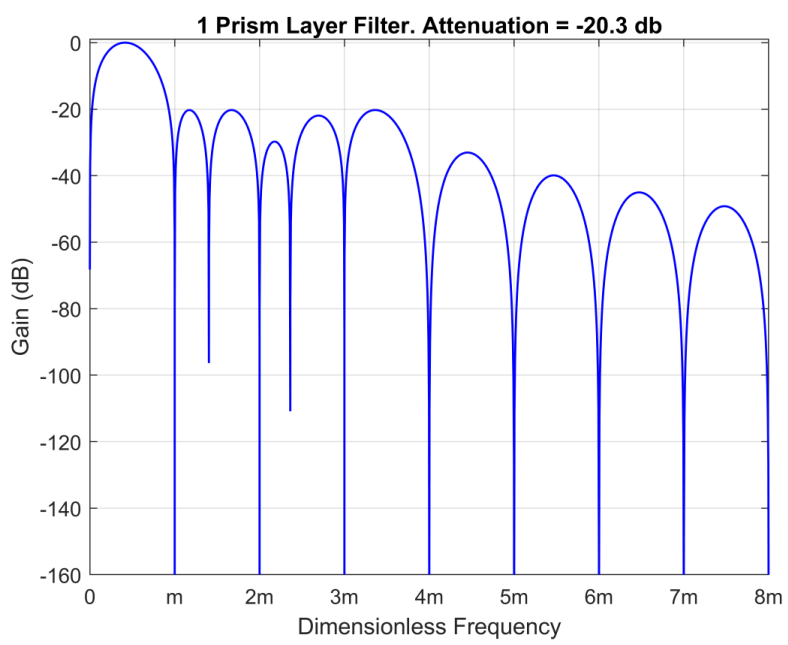

Fig. 4. Frequency response of single layer low pass Prism filter.

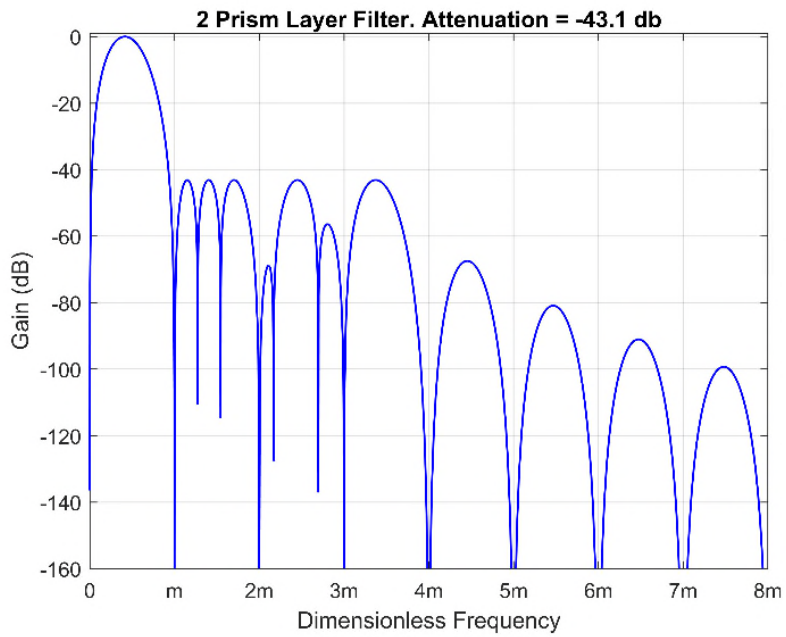

Fig. 5. Frequency response of two layer low pass Prism filter.

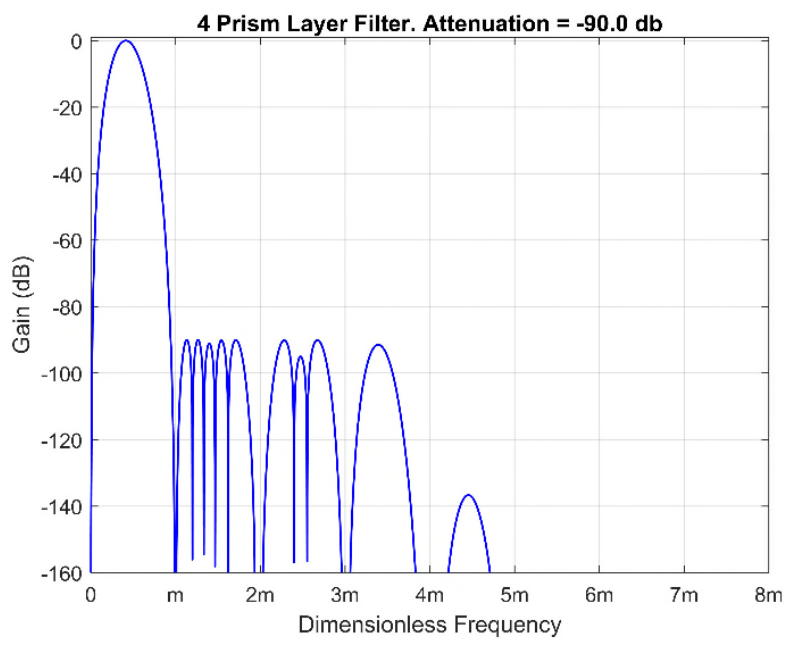

Fig. 6. Frequency response of four layer low pass Prism filter.

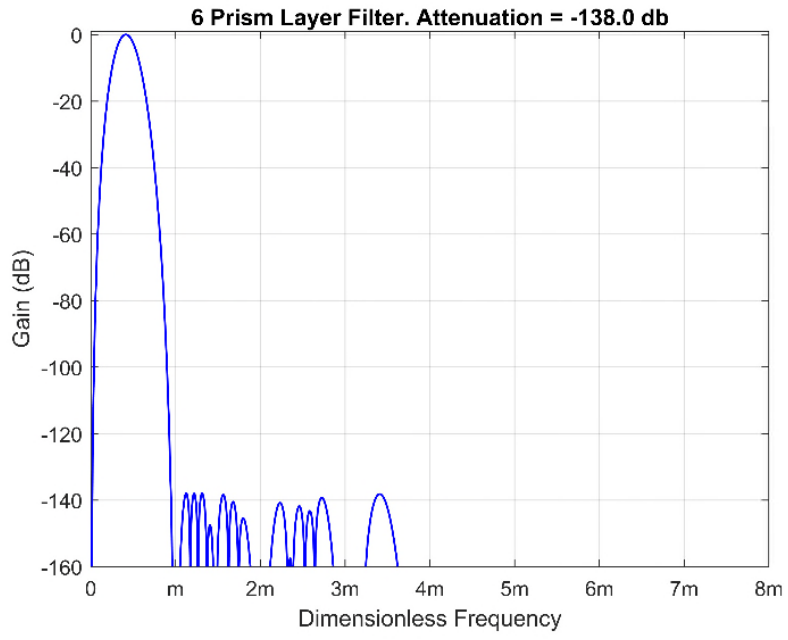

Fig. 7. Frequency response of six layer low pass Prism filter. 
With no loss of generality, it can be assumed that $w_{1}=1.0$. Optimal values are found to be $w_{2}=0.59701$ and $w_{3}=1.08407$. Fig. 4 shows the resulting frequency response, which has a maximum gain of $-20.3 \mathrm{~dB}$ for frequency above $m \mathrm{~Hz}$. The peak gain in the pass band occurs at around $0.42 \mathrm{~m}$. This low pass filter design can be instantiated simply by selecting an appropriate value of the characteristic frequency $m$.

Greater attenuation can be introducing by adding further Prism layers, where the output of each layer becomes the input to the subsequent layer. Here the values of $m$ and $w_{l}(\equiv 1.0)$ are kept constant, while unconstrained optimization is used to select the $w_{2}$ and $w_{3}$ values across all layers to maximize attenuation. Figs. $5-7$ show designs for 2, 4 and 6 layer filters, with attenuations of $-43.1 \mathrm{~dB},-90.0 \mathrm{~dB}$, and $-138.0 \mathrm{~dB}$ respectively. This greater attenuation is obtained at the cost of a higher computational burden and a slower dynamic response. As with the single layer case, the values of the $w_{i}$ are fixed: instantiation for any specific application simply requires selection of the desired value of $m$.

A key characteristic of any Prism frequency response is that all multiples of $m$, including DC, have zero gain. Accordingly, it is not possible to create a low pass filter with, for example, a flat pass band. Instead, signal processing schemes are designed so that desired frequency components are in high gain regions. As discussed in [1], simple formulae can be used to calculate the gain at any specific frequency, so that gain compensation can be applied as required.

A further characteristic of these low pass filter designs is that, by definition, the high gain region is close to DC. While Prism-based bandpass designs are described in [8,9], the utility of the low pass filter designs can be extended to higher frequency regions through the use of heterodyning, as shown in the example below.

\section{Simulation EXAMPLE}

A simulation example is provided to illustrate how a low pass filter design of this type can be used in combination with other Prism techniques to solve difficult measurement problems. Tracking a sinusoidal component in a signal can be rendered difficult by the presence of other, possibly stronger, components at adjacent frequencies, and the presence of noise.

The simulated signal contains the following components:

1. Frequency $100,000 \mathrm{~Hz}$; amplitude variable but around $1 \mathrm{mV}$; to be tracked.

2. Frequency $100,005 \mathrm{~Hz}$; fixed amplitude $100 \mathrm{mV}$; to be tracked.

3. White noise with standard deviation $1 \mathrm{mV}$; to be filtered out.

The sample rate is $500 \mathrm{kHz}$. Fig. 8 shows $30 \mathrm{~s}$ of the time series of the data (n.b. after an initial period of 20s warm up) and the power spectrum of the key spectral range. Component 1 is at $100 \mathrm{kHz}$, while Component 2, only $5 \mathrm{~Hz}$ higher, is also two orders of magnitude larger. To test the tracking ability of the solution, a step change in Component 1 is introduced at $\mathrm{t}=$ $25 \mathrm{~s}$, whereby its amplitude is reduced from $1 \mathrm{mV}$ to $0.5 \mathrm{mV}$.

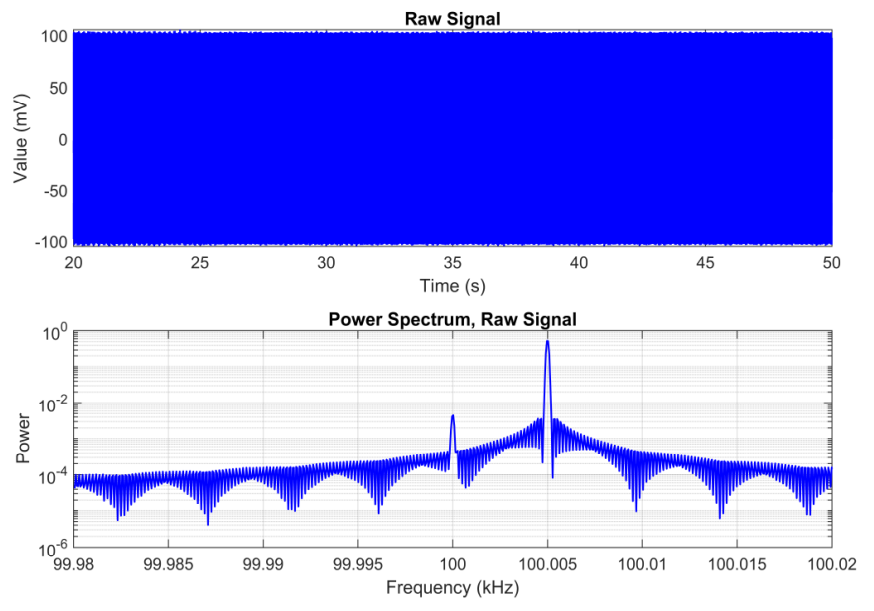

Fig. 8. Time series and frequency spectrum detail of simulation input signal.

This change is not visible in the time series, which is dominated by the $100 \mathrm{mV}$ strength of Component 2

The signal processing challenge is to track the frequency, phase and amplitude of both components, using the low pass filtering technique introduced in this paper. The same filter design is used in each case, but a different heterodyning shift is applied to select the appropriate component. Post-filtering, Prism-based tracking is used to obtain the frequency, phase and amplitude of the filtered signal each sample; has previously been described and applied in a number of instrumentation applications ([2,5]). Tracking Component 2 should be straightforward. However, tracking component 1, given the near proximity of the much larger component and a high level of white noise, represents a more significant challenge.

With only $5 \mathrm{~Hz}$ difference between the two components, a six layer filter (Fig 7) is selected to provide high stopband attenuation. The value of $m$ is chosen to be $2.4 \mathrm{~Hz}$, in order to provide good separation between the two components. Given this choice of $m$, the passband peak frequency occurs at $1 \mathrm{~Hz}$. As described in $([1,2,5])$, the tracker Prism provides two outputs, a sine/cosine pair, facilitating the calculation of sinusoidal parameters. For an expected input signal frequency of around $1 \mathrm{~Hz}$, the optimal value of $m$ for the tracker Prism is $2 \mathrm{~Hz}$.

The signal processing of both components uses the same filter and tracker design, but heterodyning is used to distinguish them as follows:

1. For Component 1 , the original signal is multiplied by a pure sinusoid with unity amplitude and a frequency of 999,999 $\mathrm{Hz}$. This has the effect of mapping the Component 1 to $1 \mathrm{~Hz}$, while Component 2 is mapped to $6 \mathrm{~Hz}$.

2. For Component 2 , the original signal is multiplied by a pure sinusoid with unity amplitude and a frequency of $1,000,004 \mathrm{~Hz}$. This has the effect of mapping the Component 2 to $1 \mathrm{~Hz}$, while Component 2 is mapped to (-) $5 \mathrm{~Hz}$.

In each case, the phase of the heterodyning signal is recorded on a sample-by-sample basis. This facilitates reconstruction of the original signal phase after tracking has been carried out on the heterodyned and filtered signal. 

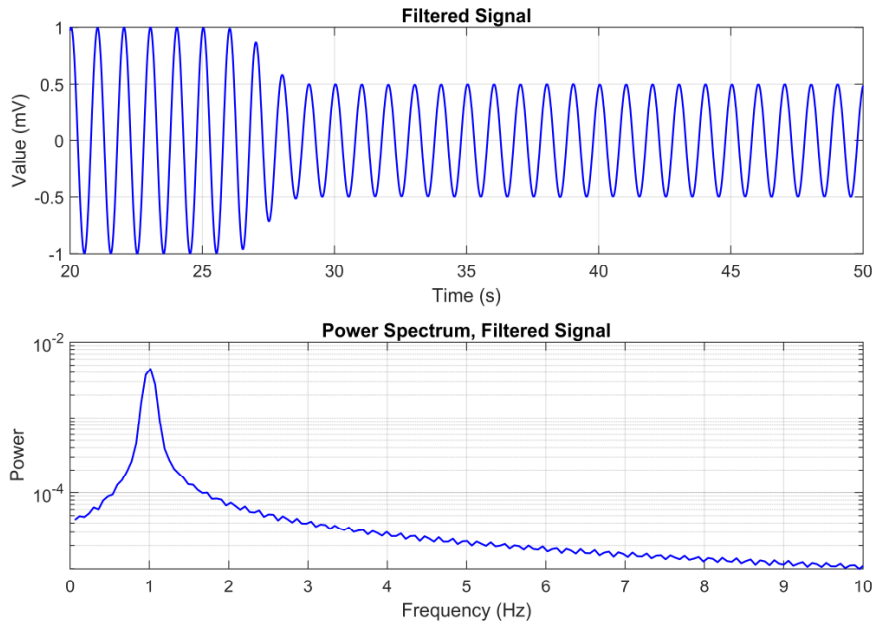

Fig. 9. Time series and power spectrum of heterodyned and filtered signal Component 1 .

The results of the signal processing steps are now presented for each component. After heterodyning, the low pass filter is applied to the signal. The results for Component 1 are given in Fig. 9. This shows the time series and spectrum of the heterodyned and filtered signal. Component 1 has been retained and positioned at the $1 \mathrm{~Hz}$ peak, while Component 2, which should be located at $6 \mathrm{~Hz}$, has been entirely attenuated by the 6 layer filter. The time series of Fig 9 shows a clean sinusoid, where the step change in amplitude, occurring at $t=25 \mathrm{~s}$, is extended over time due to the dynamic response of the bandpass filter.

Figs. $10-12$ show the tracked amplitude, frequency and phase time series calculated for Component 1. These have been obtained by applying a Prism-based tracker to the filtered signal of Fig. 9. Compensation has been applied for filter gain, (linear) filter phase delay, and heterodyning. The frequency is tracked to within $0.01 \mathrm{~Hz}$ (Fig 10). The step change in amplitude at $25 \mathrm{~s}$ results in a smooth transient response between $25 \mathrm{~s}$ and $31 \mathrm{~s}$ for amplitude tracking, accompanied by only minor perturbations in the frequency and phase tracking. Outside of this step change transition the amplitude is tracked to within $3 \mu \mathrm{V}$ and the phase to within 0.03 radians. These results are achieved despite high levels of noise and the near proximity of a much higher amplitude component in the spectrum of the original signal.

Figs. $13-16$ show the corresponding results for the larger Component 2. Fig. 13 shows the results of heterodyning and bandpass filtering. Component 2 has been mapped to the $1 \mathrm{~Hz}$ location, resulting in a steady amplitude of around $100 \mathrm{mV}$ as expected, while Component 2 , has been mapped to $(-) 6 \mathrm{~Hz}$, and has been attenuated by the filtering. The frequency (Fig. 14) is successfully tracked with a maximum error of approximately 1e-5 Hz. The amplitude (Fig. 15) is tracked with a maximum error of approximately $3 \mu \mathrm{V}$. It is unclear whether the slightly higher perturbation in amplitude around $28 \mathrm{~s}$ is due to the step change in Component 1, but its impact is marginal. The phase tracking (Fig. 16) is steady, with the error magnitude not exceeding 3e-4 radians.
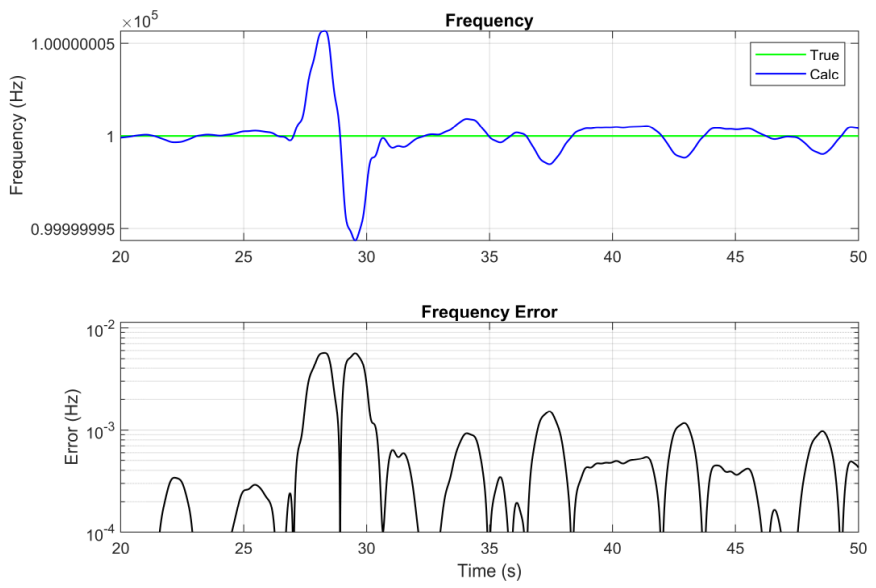

Fig. 10. Tracked frequency - Component 1
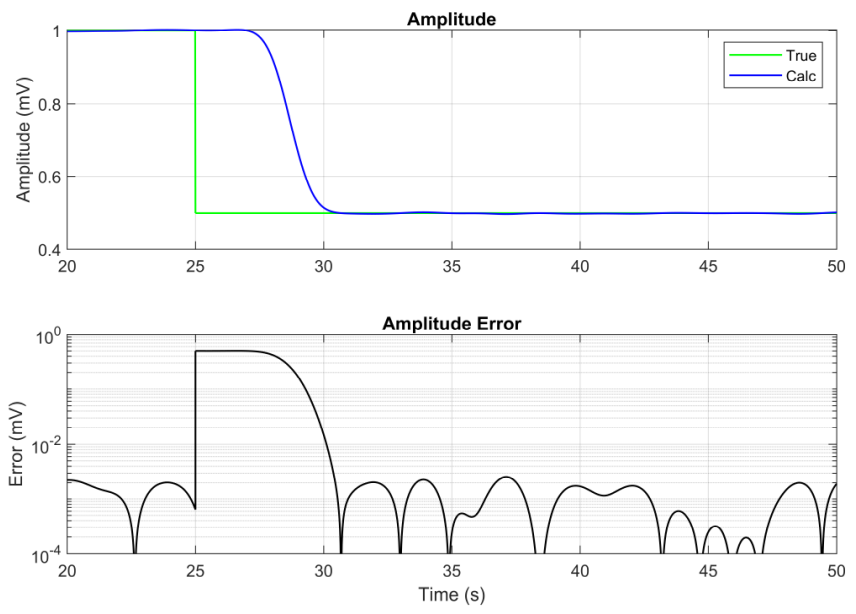

Fig. 11. Tracked amplitude-Component 1 .
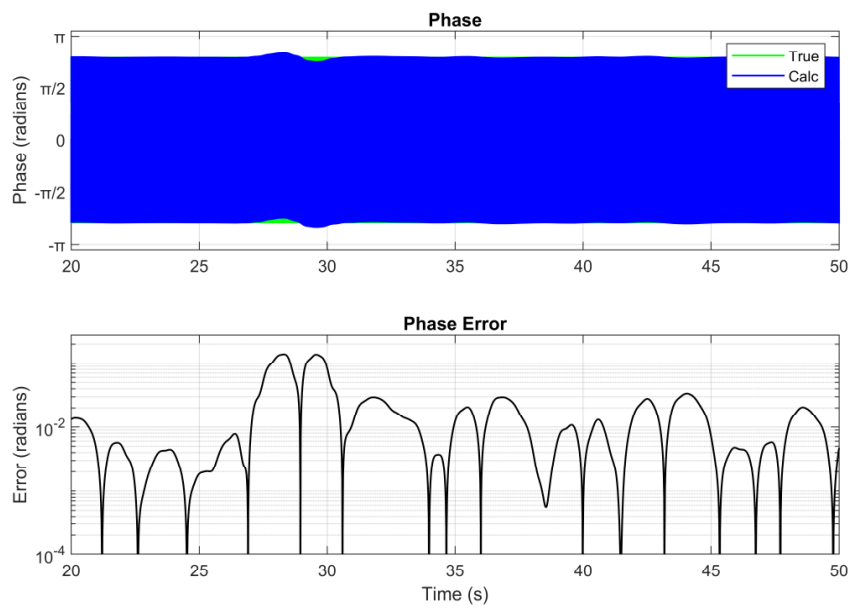

Fig. 12. Tracked phase-Component 1 

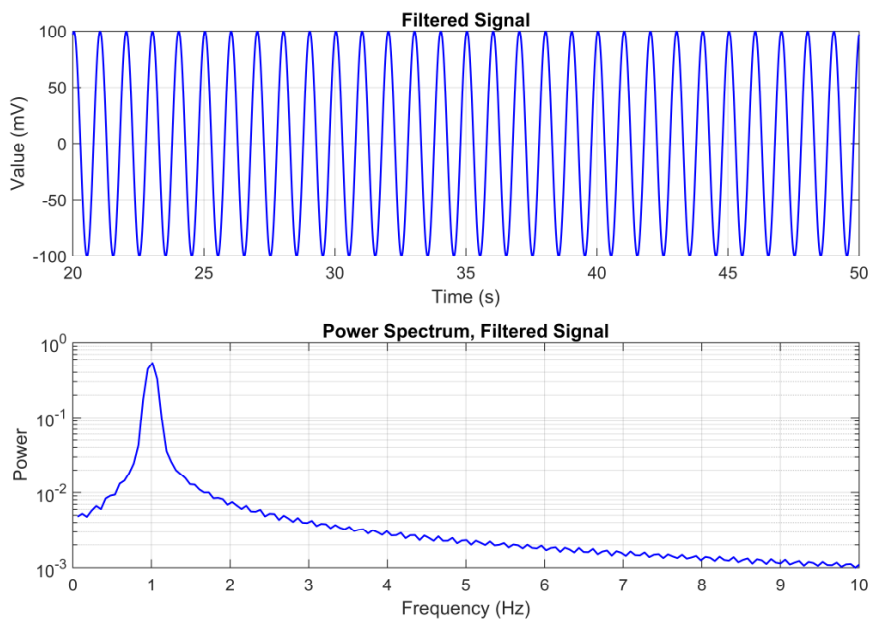

Fig. 13. Time series and power spectrum of heterodyned and filtered signal Component 2.
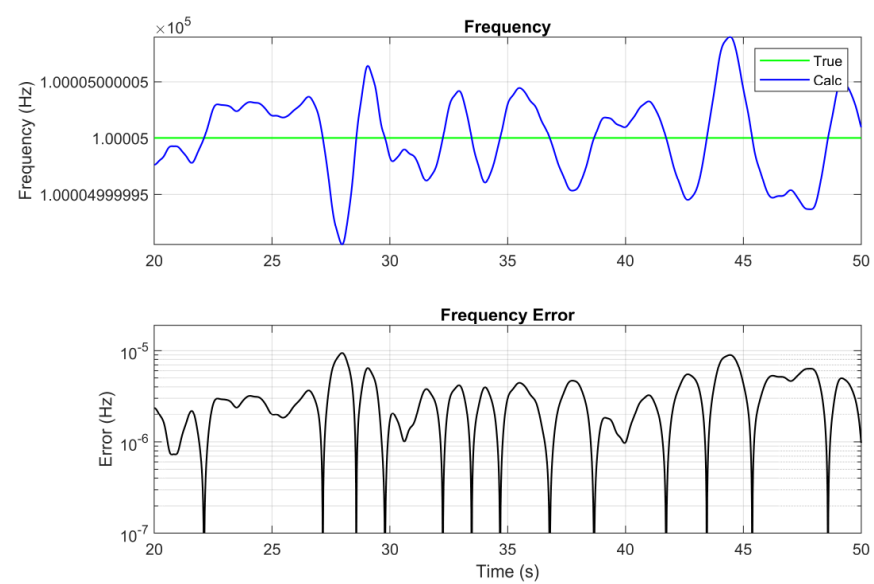

Fig. 14. Tracked frequency - Component 2
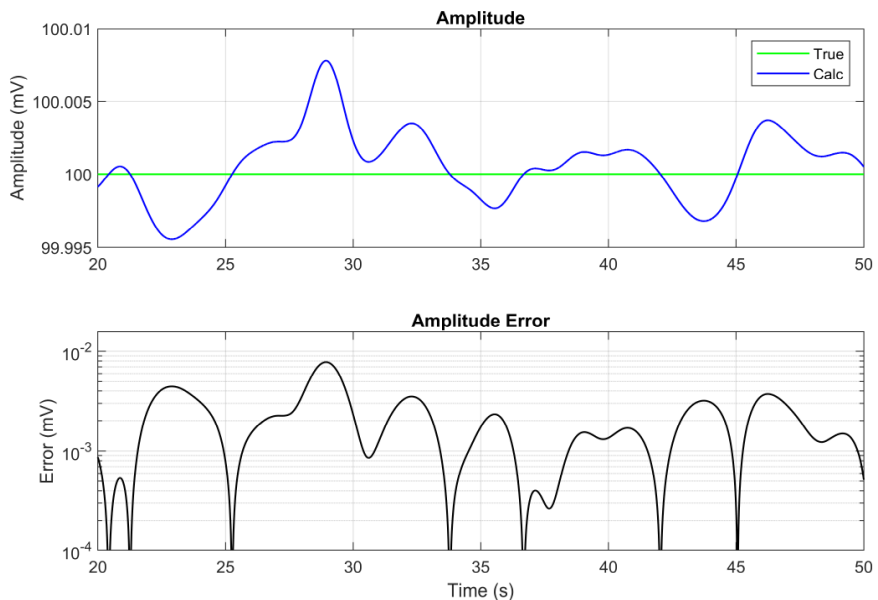

Fig. 15. Tracked amplitude - Component 2
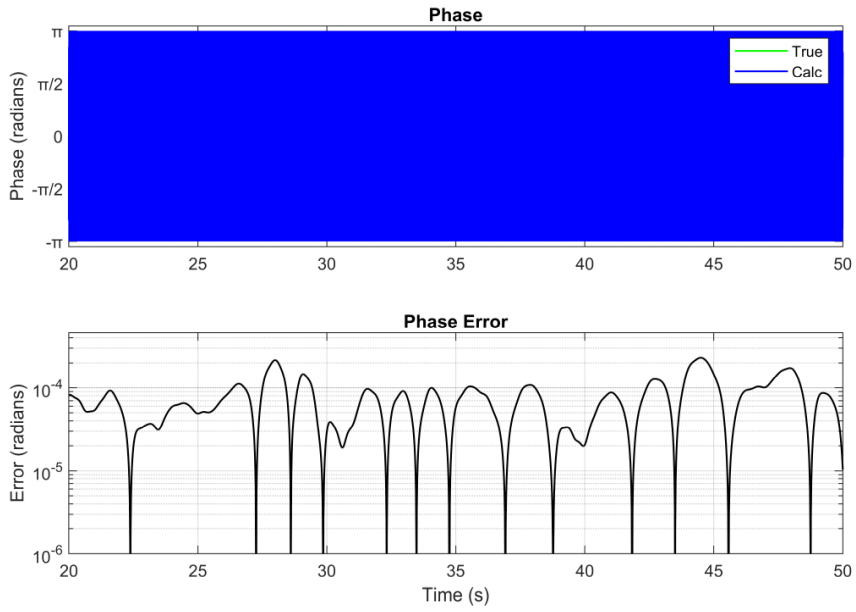

Fig. 16. Tracked phase - Component 2

\section{SUMMARY}

This paper has described a family of low pass filters created using Prism layers. These provide varying levels of stopband attenuation, with corresponding cost in terms of computational overhead and dynamic response. Their utility has been demonstrated through a simulation in which two adjacent frequency components have been separated and tracked in real time.

A key benefit of this approach is that the design and computational overheads are low, due to the recursive, nonconvolutional calculation entailed with Prism signal processing. In the context of the Internet of Things, the need for low cost, simple and robust measurement algorithms will become increasingly important: as the number of sensors deployed in the environment increases exponentially, the signal processing design effort available per sensor will decrease [2]. Signal processing design simplicity further supports the development of autonomous systems, whereby local signal processing schemes can be reconfigured in response to realtime events, for example in condition monitoring [6,7].

The low pass filtering described here will used to develop next generation instrumentation, particularly for flow measurement.

\section{REFERENCES}

[1] MP Henry. "The Prism: recursive FIR signal processing for instrumentation applications". IEEE Transactions on Instrumentation and Measurement, April 2020.

[2] MP Henry, F Leach, M Davy, O Bushuev, MS Tombs, FB Zhou, and S Karout, "The Prism: Efficient Signal Processing for the Internet of Things", IEEE Industrial Electronics Magazine, pp 2-10, December 2017.

[3] MP Henry, O Bushuev, O Ibryaeva. "Prism Signal Processing for Sensor Condition Monitoring”, 26th IEEE International Symposium on Industrial Electronics, Edinburgh, UK, 2017.

[4] F. Leach, M. Davy, M. Henry, M. Tombs, and F. Zhou, "A New Method for Measuring Fuel Flow in an Individual Injection in Real Time," in SAE Technical Papers, 2018, vol. 2018-April.

[5] Henry MP, Zhou FB, Tombs MS, Leach F, Davy, M, Malladi M. "Prism Signal Processing of Coriolis Meter Data for Gasoline Fuel Injection Monitoring”, Flow Measurement and Instrumentation, October 2019.

[6] MP Henry, V Sinitsin. "Prism Signal Processing for Machine Condition Monitoring I: Design and Simulation”, 1 st IEEE International Conference 
on Industrial Cyber-Physical Systems (ICPS-2018), May 2018.

[7] MP Henry, MP, V Sinitsin. "Prism Signal Processing for Machine Condition Monitoring II: Experimental Data and Fault Detection", 1st IEEE International Conference on Industrial Cyber-Physical Systems (ICPS-2018), May 2018

[8] MP Henry, "Ultra narrowband filtering with Prism signal processing: design and simulation," IECON, pp. 2748-2753, 2018.

[9] J. Owen, MP Henry, "384 TMAC/s FIR filtering on an Artix-7 FPGA using Prism signal processing," IECON 2018 - 44th Annu. Conf. IEEE Ind. Electron. Soc., vol. 1, pp. 2659-2664, 2018. 